\title{
El modelo pedagógico dialógico crítico en la educación
}

\section{The critical dialogic pedagogical modeling education}

\author{
Henry de Jesús Gallardo-Pérez ${ }^{1}$
}

Forma de citar: H. D. J. Gallardo. "El modelo pedagógico dialógico crítico en la educación", Respuestas, vol. 19, no. 2, pp. 81-92, 2014.

Recibido:

Noviembre 12 de 2013

Aceptado:

Abril 15 de 2014
'Doctor en Educación henrygallardo@ufps.edu.co Universidad Francisco de Paula Santander
- Colombia

\section{Resumen}

Antecedentes: La Universidad Francisco de Paula Santander en su Proyecto Pedagógico Institucional define un enfoque pedagógico dialógico crítico que tiene su origen, por una parte, en los diálogos socráticos y se basa en las teorías de Freire y Habermas, entre otros, entendiendo el diálogo como una forma de expresar el pensamiento, las acciones comunicativas como forma de generar conocimiento y la educación como un proceso que involucra a todos los actores para promover interacciones humanas que propendan por la transformación tanto de quien enseña como de quien aprende, y por otra, en la escuela de Frankfurt que sustenta la teoría crítica de la sociedad, comprendiendo la educación como un proceso que involucra a todos los actores, y tiene como propósito motivar una reflexión permanente por parte de la comunidad docente de la Universidad sobre sus prácticas pedagógicas. Objetivo: Reflexionar sobre los fundamentos ontológicos, epistemológicos, teóricos y contextuales del modelo pedagógico institucional. Métodos: Para la realización del artículo se procedió en primer lugar a la consulta de producción bibliográfica de los autores más reconocidos y la revisión de diversos artículos de investigación relacionados con el tema, y posteriormente al análisis teórico y contrastación académica con la actividad docente en la UFPS. Conclusión: Los profesores de la Universidad desarrollan prácticas pedagógicas que orienten la labor docente a un ejercicio permanente de cambio incorporando los últimos avances científicos y la actualización de contenidos temáticos contextualizados a la solución de problemas del entorno social. La docencia en la UFPS no es una actividad reducida a la enseñanza en el aula, es todo un sistema que a su vez está constituido por una serie de subsistemas, que interactúan para contribuir al mejoramiento continuo de su calidad y productividad en un proceso continuo de apropiación por parte de los docentes del enfoque pedagógico dialógico-crítico en los procesos de formación de sus estudiantes.

Palabras clave: Crítico-Dialógico, Educación, Modelo Pedagógico. 


\begin{abstract}
Background: The Francisco de Paula Santander University in its Institutional Education Project defines a critical dialogical pedagogical focusing that originates, first, in the Socratic dialogues and is based on the theories of Freire and Habermas, among others, understanding the dialogue as a way of expressing the thought, communicative actions as a way of generating knowledge and education as a process that involves all stakeholders to promote human interactions which foster the transformation of both who taught as part of the learner, and secondly, in the Frankfurt School critical theory underpinning of society, comprising education as a process involving all stakeholders, and aims to encourage ongoing reflection by the faculty of the University community about their pedagogical practices. Objective: Reflecting on the ontological, epistemological, theoretical and institutional context of the pedagogical model. Methods: To achieve the article was first proceeded to query bibliographic production of the most renowned authors and review of various research articles related to the topic, and then the theoretical analysis and academic teaching activities contrasting with the UFPS. Conclusion: University teachers develop pedagogical practices that orient teaching activity in a permanent change incorporating the latest scientific advances and updating content areas contextualized problem solving social environment. Teaching in the UFPS is not reduced to teaching in the classroom activity, it is an entire system which in turn consists of a number of subsystems, which interact to contribute to the continuous improvement of quality and productivity of teaching in a continuous process appropriation by teachers of critical dialogical processes of formation of its students pedagogical approach.
\end{abstract}

Keywords: Critical-Dialogic, Education, Pedagogical Model.

\section{Introducción}

La Universidad Francisco de Paula Santander

82 (UFPS) es una Institución pública de Educación Superior del orden Departamental, cuyo propósito fundamental es la formación integral de profesionales, comprometidos con la solución de problemas del entorno, en busca del desarrollo sostenible de la región. El Proyecto Educativo Institucional (PEI) propone un enfoque pedagógico dialógico y crítico, centrado en la construcción del conocimiento a partir del diálogo y la búsqueda del conocimiento entre el maestro y sus estudiantes.
La definición de un enfoque crítico y dialógico en la UFPS es el resultado de los foros académicos que dieron origen al proyecto educativo institucional en el año 2007. Su principal objetivo es abrir el debate en torno a las prácticas docentes en la Universidad y proponer una ruta pedagógica que incorpore la investigación, el constructivismo y la formación por competencias, como posibilidad para la excelencia académica. Constituyen este artículo, cuatro acápites que permiten definir el modelo desde los puntos de vista ontológico, epistemológico, teórico y contextual, tanto para el enfoque dialógico como para su componente crítico. 
En modelo pedagógico de la UFPS se concibe como un sistema que interrelaciona a los agentes de su comunidad educativa con el conocimiento científico (De Zubiría, 1994), mediante la representación de las acciones, actividades y procedimientos que predominan en los procesos de enseñanza y de aprendizaje, propiciando el desarrollo de las potencialidades de los estudiantes, de los profesores y de las ciencias, para establecer un diálogo y crítica permanentes entre docentes y estudiantes en un ambiente de crecimiento académico con el fin de alcanzar tanto un aprendizaje significativo como el fortalecimiento de competencias con proyección a mejorar el entorno social y la calidad de vida (UFPS, 2006).

El enfoque pedagógico dialógico-crítico adoptado por la UFPS en su Proyecto Educativo Institucional, tiene como propósito motivar una reflexión permanente por parte de la comunidad docente de la Universidad sobre sus prácticas pedagógicas (UFPS, 2006) y adopta como principio institucional:

Se propone un enfoque pedagógico, dialógico y crítico, desde una perspectiva que asuma la práctica pedagógica como una cultura de paz; por lo tanto, se generarán políticas académicas que contribuyan a erradicar el simple transmisionismo de información y se privilegien aquellos modelos pedagógicos centrados en la construcción del conocimiento, a partir del diálogo permanente entre el maestro y su estudiante en torno a la ciencia, atendiendo la formación integral de nuestros profesionales (p. 26)

Es decir, una pedagogía que forme y transforme a las nuevas generaciones a partir de reconocer la potencialidad y responsabilidad para desarrollar la creatividad, la imaginación y la inteligencia a partir del estímulo a la crítica de las ideas y el diálogo fundado en la tolerancia. El modelo está basado en una filosofía humanista - empirista con énfasis en propiciar el diálogo y las relaciones interpersonales, motivando el aprendizaje contextualizado a partir de contenidos problémicos, con el fin de desarrollar la capacidad de interpretación del alumno mediante el trabajo en grupo y desarrollo de experiencias de aprendizaje, evaluando logros con enfoque cualitativo.

El enfoque pedagógico de la UFPS tiene su origen en los diálogos socráticos, cuya experiencia pedagógica se centraba en el diálogo y en la pregunta del maestro Sócrates a sus discípulos y está basado en la "Teoría de la Acción Dialógica" de Freire (1970) para quien la naturaleza humana es dialógica y la comunicación constituye un papel fundamental en nuestra vida, la aproximación de la indagación dialógica como un enfoque educativo que reconoce la relación dialéctica entre el individuo y la sociedad, y una actitud para la adquisición de conocimientos a través de las interacciones comunicativas de Wells (2001), con la teoría de la Acción Comunicativa de Habermas (1987) en la que el conocimiento es el proporcionado por el mundo objetivo y por la intersubjetividad del contexto donde se desarrolla la acción, la noción de la imaginación dialógica de Bakhtin (1981) según la cual existe la necesidad de creación de significados de una manera dialógica con otras personas y establece una relación entre el lenguaje, la interacción y la transformación social y la teoría del "Yo dialógico" de Soler (2004).

Este enfoque pretende centrar las prácticas pedagógicas desde el aprendizaje de los estudiantes, lo cual obliga una autoevaluación permanentemente de la manera como los docentes entienden el proceso de aprender de sus alumnos, llevando a plantear la práctica docente de modo que estimule la construcción de conocimiento y no la simple transmisión de datos y contenidos, Urbina (2010); requiere por tanto del trabajo conjunto de docentes para estimular tanto el aprendizaje colaborativo como para establecer planes de formación que estimulen el diálogo y la crítica no sólo en sus asignaturas sino mostrando complementariedad en la formación de estudiantes mediante el desarrollo de proyectos conjuntos.
Julio-Diciembre 2014 ISSN 0122-820X

PP: 81-92 
No. 2

Julio-Diciembre 2014 ISSN 0122-820X

PP: 81-92

\section{Fundamentos teóricos del modelo}

El modelo pedagógico tiene sus bases en el pensar griego, en particular en la filosofía de Platón (1972), quien elige el diálogo como forma de expresar su pensamiento poniendo a Sócrates como su interlocutor. Platón (1972) critica las explicaciones del conocimiento dadas por los filósofos que lo precedieron negando afirmaciones como que el conocimiento se pueda identificar con la percepción sensible ya que la verdad se expresa en el juicio y no en la sensación, cree por el contrario, que hay otra forma de conocimiento propia de la razón y que se dirige a un objeto distinto del objeto que nos presenta la sensibilidad: las ideas. Afirma que el verdadero conocimiento ha de versar sobre el ser, no sobre el devenir, y no puede estar sometido a error, ha de ser infalible, Calvo (1986).

En el mismo sentido, se han encontrado antecedentes en la cultura hindú en donde se pone de manifiesto el hábito de hacer preguntas con el fin de construir democracia a partir del razonamiento y el diálogo; la cultura es importante para el desarrollo, pero la libertad es primordial para la cultura, en especial, la libertad para decidir lo que se debe valorar y qué clase de vida se desea buscar, luego la educación es importante no sólo por su contribución al crecimiento económico sino por ser parte esencial del desarrollo cultural (Sen, 2007).

También se encuentran bases en la teoría de la acción dialógica de Freire (1979) en que establece que la naturaleza del ser humano es dialógica y cree que la comunicación tiene un rol principal en su vida; unos con otros dialogan continuamente y por ello una opción que favorece la labor docente es el diálogo permanente con sus estudiantes, promoviendo el entendimiento, la creación cultural y la liberación.

Refiriéndose a la educación Freire (1990) señala que como toda práctica humana lleva implícita una concepción de ser humano y de mundo, entonces toda práctica educativa por parte del educador debe llevar una posición teórica que implica una interpretación del hombre y del mundo. Sugiere el proceso comunicativo como vía para liberarse de la enajenación, es decir, para romper con la relación de opresión de unos seres humanos sobre otros. En este proceso de comunicación, cada ser humano puede expresar su palabra libre y críticamente, asumiendo con responsabilidad su participación en los procesos sociales; luego la posibilidad de ser persona esta cimentada en la posibilidad de decidir por sí mismo, que sólo puede lograrse con una educación que le permita desarrollarse como tal, ya que mientras existan relaciones de dominación habrá deshumanización. Para efectuar esta liberación Freire (1979) propone emerger de ella y liberarse de su fuerza lo cual sólo es posible hacerlo a través de la praxis auténtica; que no es ni activismo ni verbalismo, sino acción y reflexión. Para Freire (1990) el acto de conocer es un acto de estudio, y estudiar significa disciplina, sistematicidad, conciencia; estudiar es una forma de reinventar, de recrear, de rescribir, y es una tarea del sujeto más no del objeto; por tanto, conocer, que es siempre un proceso, supone una acción dialógica.

Para Feuerbach (1983), a quien se le atribuye el descubrimiento del carácter comunicativo del individuo, el ser humano es individuo y género al mismo tiempo. Un individuo sin sociedad no es humano por cuanto no puede asumir la identidad de género; un ser social sin cuerpo y mente individuales no es humano. Señala que desde su nacimiento el ser humano es configurado por los otros de modo que su naturaleza se realiza y se completa en esa multiplicidad de voces que construye su específica existencia: por tanto, es el fruto de una red de comunicación que comienza cuando la persona nace y va hasta su fallecimiento. Propone un ser humano ontológicamente comunicativo que define su personalidad humana gracias a su relación dinámica y dialógica con otros individuos del mismo género $y$, en mayor medida, gracias a la intuición sensible del tú, como concepto de otredad, Cabedo (2000). 
La teoría de la Acción Comunicativa desarrollada por Habermas (1987) implica que la construcción del conocimiento es un evento conversacional que se construye en el actuar, y que por lo tanto, debe estar alimentada por un proceso argumentativo y crítico en las cuales las pretensiones de validez del conocimiento sean cuestionadas de forma permanente y no incorporadas de manera acrítica. El concepto de acción comunicativa se refiere a la interacción de al menos dos actores capaces de desarrollar un lenguaje y de acción que, ya sea con medios verbales o extraverbales, establecen una relación interpersonal; en este contexto, los actores buscan entenderse sobre una situación de acción para poder así coordinar de común acuerdo sus planes de acción y con ello sus acciones. El autor deriva este concepto de los diversos tipos de acción que distinguió Max Weber (1996): racional, orientada por valores, afectiva y acción tradicional. Al redefinir los tipos weberianos, coloca frente a la acción instrumental la acción comunicativa como una relación interpersonal lingüística que busca el mutuo entendimiento, el consenso. Mientras en Marx (1867) la acción y la racionalidad instrumental se relacionan con el trabajo, la acción y la racionalidad comunicativa se relacionan con la interacción. Cuando la acción comunicativa se basa en argumentaciones racionales y tiene pretensiones de universalidad se denomina discurso.

Not (1962) propone distintos modelos de relación maestro estudiante, conocidos como (a) enseñanza en primera persona, que responsabiliza del éxito del aprendizaje al alumno mismo; (b) enseñanza en tercera persona, donde la responsabilidad del aprendizaje del alumno recae sobre el enseñante, el maestro es quien posee la información y el conocimiento y el alumno carece de ellos, y (c) enseñanza en segunda persona o enseñanza dialogante en donde la responsabilidad del aprendizaje surge de la interacción entre aprendizaje y aprendiz. Por tanto, en un modelo dialogante la educación se centra en el desarrollo y no en el aprendizaje, hay un reconocimiento al trabajo de las dimensiones cognitiva, socioafectiva y práxica, hay una diversidad de estrategias que garantizan la reflexión, el aprendizaje y el diálogo, y el aprehendizaje se reconoce como un proceso activo y mediado. En este modelo, la educación se concibe como un proceso en que tanto docentes como estudiantes cumplen papeles esenciales pero diferenciados; hay un direccionamiento del docente pero respetando dinámicas y procesos propios y activos del estudiante.

Julián de Zubiría (2006) afirma que como la inteligencia es diversa, la escuela no puede centrarse en una sola dimensión; también afirma que todo proceso humano es social, contextual e histórico, por tanto en la educación no se puede dejar de lado el pensar en a quién se está educando, y que las dimensiones cognitiva, socio-afectiva y práxica del ser humano son susceptibles de modificación positiva o negativa. Con base en ello propone los siguientes principios para una pedagogía dialogante: (a) el fin de la educación no debe ser el aprendizaje sino el desarrollo, (b) toda representación mental es producto de la interacción activa e interestructurante del sujeto y el medio luego la educación debe reconocer el papel activo tanto del mediador como del estudiante, y (c) sugiere la conveniencia y necesidad de trabajar por competencias, entendiéndolas como aprehendizajes de carácter integral, general y contextual que se expresan en relaciones idóneas y promueven el desarrollo de los estudiantes a nivel cognitivo, valorativo y praxológico. En consecuencia, para desarrollar un modelo pedagógico dialogante la acción educativa se debe orientar hacia la humanización, los contenidos a trabajar deben estar orientados al desarrollo de competencias, la estrategia metodológica debe desarrollarse desde un enfoque dialogante donde el estudiante juegue un papel activo y la evaluación debe abordar las tres dimensiones humanas.

Los modelos fundamentan una particular relación entre el maestro, el saber y el alumno estableciendo las principales características y
Julio-Diciembre 2014 ISSN 0122-820X PP: 81-92 
No. 2

Julio-Diciembre 2014 ISSN 0122-820X

PP: 81-92 niveles de jerarquización y delimitan la función de los recursos didácticos que se requieren para llevar a cabo su implementación mediante la representación de las acciones, actividades y procedimientos que predominan en los procesos de enseñanza y de aprendizaje, De Zubiría (2007); desde esta óptica se presentan a continuación los referentes teóricos de los componentes dialógico y crítico que fundamentan el modelo pedagógico de la UFPS.

El modelo dialógico de la pedagogía enfatiza la importancia de la acción educativa colectiva mediatizadaporlacomunicación que se obtiene como resultado de la interacción dialógica entre todas las personas que participan en las instancias educativas o que, sin participar, son esenciales para el aprendizaje; se orienta hacia la igualdad de las diferencias afirmando que la verdadera igualdad respeta las diferencias, así asegura que todas las personas, desde su cultura, religión, género y estilo de vida, entre otras, tengan las mismas oportunidades a la vez que se fomenta la igualdad de resultados, Ferrada (2008), lo cual implica que educador y educando intercambien activa y reflexivamente sus conocimientos permitiendo que se construya, desde la práctica y la realidad de los educandos, el conocimiento y la reflexión crítica del mundo, Mirabal (2008).

En el modelo dialógico, el concepto de educación se comprende como un proceso que involucra a todos los actores para promover interacciones humanas que propendan por la transformación tanto de quien enseña como de quien aprende; por tanto, se asume que toda persona es capaz de agenciar cambios al interactuar con otras pues está dotada de la acción, luego es agente-actuante, constructora y transformadora tanto de sí misma como del medio en el cual se desarrolla y vive para consolidar una sociedad dialógica.

El aprendizaje dialógico apunta a la transformación, y no a la adaptación; tiene una base interdisciplinar enmarcada dentro del paradigma social de la enseñanza, las interacciones dialógicas son fundamentales para el aprendizaje en que el lenguaje cumple un papel de mediador, destacando también el vínculo inseparable entre el individuo y la sociedad, por tanto, bajo este modelo, el aprendizaje no puede separarse del entorno social, económico y cultural en el cual está inmerso el proceso educativo y que es transformado a través del diálogo; por otra parte, en la pedagogía crítica, el estudiante es el constructor del conocimiento haciendo éste inteligible la realidad y reflexionando de manera crítica en torno a ella.

El modelo dialógico propone que los seres humanos aprenden no sólo en el contexto del salón de clase, sino en el medio en que se desenvuelven. Freire (1979), considera que el ser humano es por naturaleza dialógico y que la comunicación pedagógica es la tarea fundamental de un buen maestro; el diálogo, que es siempre comunicación, soporta la colaboración como característica de la acción dialógica que se da sólo entre sujetos en niveles distintos de función y responsabilidad sólo puede darse en la comunicación. Wells (2001) define la indagación, no como un método, sino como una predisposición a cuestionar; propone la existencia de una relación dialéctica entre el estudiante, el contexto en que vive y las tendencias de su profesión, que lo predispone al cuestionamiento permanente de los saberes que expone el maestro.

El diálogo ha jugado un papel importante en las relaciones sociales el trabajo y, en particular, en el escuela, en éstos últimos aparecen los equipos de trabajo, que no son otra cosa que grupos que interactúan buscando un objetivo común, en los cuales las decisiones son en varias ocasiones alcanzadas mediante consensos de opiniones de sus integrantes; también han surgido grupos de amigos que realizan continuamente encuentros personales y virtuales a través de las redes sociales. Estos giros afectan la escuela y la educación, los estudiantes participan de manera activa en la construcción de su propio conocimiento, la gestión institucional y la concreción de los contenidos curriculares 
mediante la interacción, el diálogo y la crítica constructiva con sus compañeros de grupos, docentes, directivos y comunidad educativa de su contexto social, llevando a que realmente en el proceso de enseñanza se trabajen problemas que realmente son de interés para los estudiantes y responden directamente a sus demandas, Díez (2000).

Por otra parte, el componente crítico del modelo de la UFPS se sustenta en la Escuela de la Teoría Crítica de la Sociedad propuesta por los teóricos críticos de la escuela de Frankfurt, entre los cuales se destacan Adorno, Horkheimer y Marcuse, quienes a mediados del siglo XX desarrollaron una perspectiva teórica y epistemológica en la cual cuestionaron la forma tradicional de hacer ciencia y acceder al conocimiento, Urbina (2010); también se soporta en los fundamentos de la pedagogía crítica expuestos por Paulo Freire, Peter McLaren, Henry Girox y Spephen Kemmis, Cervantes (2009).

La filosofía de Adorno, al igual que la de todos los exponentes de la teoría crítica, se enmarca en la corriente básica hegelianomarxista. Según Adorno (1984), la sociedad industrializada presenta una estructura que niega al pensamiento su tarea más genuina: la tarea crítica. Por tanto, la filosofía toma importancia como pensamiento crítico para disipar la apariencia de libertad, mostrar la cosificación reinante y crear una conciencia progresiva.

Horkheimer (2003) establece una distinción entre dos concepciones diferentes de "teoría": (a) La teoría tradicional que considera a su objeto de estudio como un conjunto de facticidades y al sujeto como un elemento pasivo en el acto del conocimiento, hace referencia a un conjunto de proposiciones cuya validez radica en su correspondencia con un objeto ya constituido previamente al acto de su representación convirtiendo a la teoría en una actividad pura del pensamiento y al teórico en un espectador que se limita a describir el mundo como es; y (b) la teoría crítica, en oposición a la anterior, considera que tanto la ciencia como la realidad estudiada por ésta, son un producto de la praxis social, lo cual significa que el sujeto y el objeto del conocimiento se encuentran preformados socialmente; el sujeto y objeto, son resultado de procesos sociales muy complejos, así, la teoría se constituye en un elemento de la praxis social.

La educación problematizadora propuesta por Freire (1979), en lugar de lo que él denomina educación bancaria (acumuladora de información), busca liberar el pensar mediante la acción de los seres humanos en su tarea de rehacer el mundo y transformarlo en uno más humano. Freire considera la pedagogía como instrumento de lucha por la liberación de los oprimidos y la educación como la generación de una conciencia crítica.

Para McLaren (1995), el objetivo de la pedagogía crítica es habilitar a los desposeídos y trasformar las desigualdades e injusticias sociales existentes. Es una pedagogía de la liberación y de la esperanza que lucha por una vida cualitativamente mejor para todos mediante la construcción de una sociedad basada en relaciones no explotadoras y en la justicia social. Considera las escuelas como espacios para la transformación social y la emancipación, son arenas culturales donde una heterogeneidad de formas sociales e ideológicas suelen enfrentarse en una lucha irremisible por la dominación que poseen una doble forma: como mecanismos de clasificación y como agencias para dar poder, Góngora (2008).

Ahora bien, para Giroux (1989), las escuelas son agencias de reforma social que educan a ciudadanos capaces de construir una democracia crítica; por tanto, los maestros requieren una formación que promueva una nueva visión de la escuela y la enseñanza en un concepto de ciudadanía crítica, luego deben trabajar como investigadores reflexivos y como intelectuales transformadores en el contexto de la comunidad donde se hallan inmersos, entendiendo el salón de clases como un terreno cultural, neutral y transparente en
Julio-Diciembre 2014 ISSN 0122-820X

PP: 81-92 
No. 2

Julio-Diciembre 2014 ISSN 0122-820X

PP: 81-92 donde se deben diferenciar las experiencias, conocimientos y rasgos culturales de los alumnos.

Las comunidades críticas, según Kemmis (2007), nacen de la necesidad de contrarrestar la reducción de la educación a los meros valores instrumentales y para mantener en pie los valores de la sociedad educativa, su propósito es la reflexión, el autoconocimiento y la acción política eficaz usando como método el diálogo y la investigación educativa; pueden construirse en una gran variedad de ambientes y en ellas participan estudiantes, profesores, padres, administradores escolares y otras personas interesadas, Areyuna (sf.). Para que exista una comunidad crítica, se requiere que las personas compartan creencias y valores y que las relaciones sean directas, múltiples y se caractericen por una reciprocidad equilibrada existiendo un sentimiento de solidaridad, fraternidad y respeto mutuo. Los principales resultados de esta acción pedagógica conllevan a elevar la autoconciencia y el potencial de acción colectiva de los sujetos participantes.

La pedagogía crítica cuestiona la realidad educativa al tiempo que asume una participación social que permita la contextualización de los procesos educativos, para que mediante la interacción entre el profesor, los estudiantes y la sociedad en torno al saber se alcance una transformación de esa realidad social, lo cual incluye enseñar a toda la comunidad educativa a aprender de su propia práctica; propone las relaciones maestroalumno como una etapa necesaria para el desarrollo del capital humano y la focaliza en la formación de profesionales creativos, con autonomía, capaces de identificar problemas de su entorno y de plantear alternativas de solución construyendo consensos de manera dialógica en interacción con los agentes del medio. Como lo expresa McLaren (1995), la pedagogía crítica es una política de entendimiento y un acto de saber que intenta situar la vida cotidiana en un contexto geopolítico, con la meta de estimular una auto-responsabilidad colectiva regional, un ecumenismo a gran escala y una solidaridad internacional de los trabajadores

Las principales características de la teoría crítica son: (a) Negatividad: La dialéctica negativa propuesta por Adorno (1966) afirma que no todo lo real es totalmente racional, adquiriendo relevancia la dialéctica positiva que requiere al sujeto adecuarse a la realidad e incluso someterse a ella en la práctica haciendo eterno el instante presente e inhibiendo cualquier acción transformadora (revolucionaria); se rechaza la posibilidad de construir una utopía positiva en tanto no es posible determinar cómo habría de ser el futuro, pero sí es posible establecer como "no debe ser", lo cual es suficiente para poder criticar el presente; (b) Mediación: La teoría crítica afirma que la producción teórica no puede existir independientemente de los procesos socio-históricos y económicos dentro de los cuales ha surgido y por tanto todo conocimiento está determinado por mediaciones, siendo el contexto histórico quien determina el objeto y la finalidad de toda investigación, en contraposición con la teoría tradicional que pretende la inmediatez entre el sujeto y el objeto; (c) Praxis: para Marcuse (1993), el problema de la objetividad histórica requiere juicios de valor, lo que implica estar al servicio de la emancipación humana y derivar en una praxis liberadora. En síntesis, los dos polos de la teoría crítica son razón y praxis.

\section{Fundamentos contextuales del modelo}

La contextualización del enfoque pedagógico dialógico crítico adoptado por la UFPS en su PEI tiene como propósito motivar la reflexión permanente por la comunidad docente sobre sus prácticas pedagógicas centrándolas desde el aprendizaje de sus estudiantes con el fin de que la preocupación no se relacione tanto con la enseñanza sino que se enfoque sobre el aprendizaje y las competencias cognitivas, emocionales, actitudinales y la forma como los estudiantes utilizan el saber para enfrentar los retos de la sociedad del conocimiento y los nuevos problemas del mundo globalizado, estimulando la construcción del conocimiento 
en sus estudiantes (Urbina, 2010). El modelo pedagógico adoptado por la Universidad lleva implícita una re-contextualización para la educación superior del modelo pedagógico contemporáneo (De Zubiría, 2007), manifiesta una complementariedad de los modelos desarrollista y socio-crítico (Flórez, 2005) y el modelo pedagógico integrado propuesto por Mario Díaz (2003, 2009).

Se espera que cualquier profesor de la Universidad pueda desarrollar el enfoque a partir de prácticas sencillas como las siguientes: (a) estimular la pregunta en lugar de las respuestas, (b) crear ambientes de aprendizaje que favorezcan la participación de los estudiantes en la resolución de problemas, (c) orientar las clases hacia la realización de proyectos sencillos y aplicables por parte de los estudiantes, (d) estimular el trabajo cooperativo, (e) motivar el uso de TIC como estrategia para profundizar y $(\mathrm{f})$ orientar las evaluaciones hacia la solución de problemas y no hacia la respuesta repetitiva de contenidos mecánicos. Este tipo de prácticas modifican la costumbre de pretender "dictar clase", entendiéndose esta como la repetición de temas los estudiantes los memoricen y los repitan, orientando la labor docente a un ejercicio permanente de cambio de la docencia a partir del interrogante surgido sobre cómo aprenden los alumnos y las estrategias pedagógicas a desarrollar para lograr un aprendizaje significativo, la realización de clases interesantes, la incorporación de los últimos avances científicos y actualización permanente de contenidos temáticos y su contextualización a la solución de problemas del entorno social.

Al indagar sobre la aplicabilidad del modelo en la UFPS, Gallardo (2013) encuentra, mediante encuesta de pregunta abierta aplicada a una muestra aleatoria de estratificada aplicada al $10 \%$ de los docentes de la Institución que el $70 \%$ de los docentes manifiesta la realización de talleres en sus clases; hay un $60 \%$ que realiza clases magistrales complementadas con otras actividades de formación; un 55\% plantea problemas o situaciones problémicas en sus clases así como ejercicios de aplicación de conceptos; también se encuentra un $55 \%$ que realiza prácticas de laboratorio, incluyendo los de ciencias básicas, de computación, de electrónica, de mecánica y otros de ingeniería, los laboratorios empresariales, laboratorios especializados y prácticas de campo; solamente el 35\% de los docentes manifiesta utilizar las nuevas tecnologías de información y comunicación en sus actividades académicas; también un 35\% realiza actividades de investigación formativa y otro 35\% exposiciones orientadas; alrededor del 25\% elaboran guías de trabajo; hay un $20 \%$ de docentes que involucran actividades de aprendizaje colaborativo y otro $20 \%$ que utiliza la construcción de consensos en los procesos de formación de sus estudiantes.

También se encuentra en este estudio que tres de cada cuatro docentes de la UFPS implementan dentro de su cátedra la investigación formativa, entendiéndose ésta como la capacidad para desarrollar en el estudiante el pensamiento lógico, la facultad para formular preguntas que sean susceptibles de convertirse en problemas de investigación y el planteamiento de estrategias que permitan proponer soluciones a estos problemas concretos; las actividades de formación más referidas a este aspecto fueron: la investigación bibliográfica, incorporación de casos que vinculen la teoría con la práctica, relación de los conocimientos y métodos propios de la disciplina con el contexto regional, profundización en el resultado de prácticas de laboratorio, profundización en aplicación de leyes y teorías, participación en seminarios y conformación de semilleros de investigación; estas actividades son consideradas como básicas para desarrollar proyectos de investigación relacionados con los contenidos temáticos del curso

Para indagar sobre el conocimiento que se tiene sobre el enfoque pedagógico críticodialógico de la Universidad, se preguntó sobre qué se entiende por este enfoque pedagógico; el 34\% dejó sin respuesta este ítem o expresó una definición no correcta, lo cual lleva a
Julio-Diciembre 2014 ISSN 0122-820X

PP: 81-92 
No. 2

Julio-Diciembre 2014 ISSN 0122-820X

PP: 81-92 la conclusión que no lo conoce; del $66 \%$ restante que expresó opiniones sobre el enfoque, de los cuales el $63 \%$ lo define de una manera bastante bien, el 25\% tiene una idea aproximada del enfoque y el $12 \%$ expresa opiniones algo relacionadas con la concepción de este enfoque.

Finalmente, el análisis de las respuestas relacionadas con el enfoque permitió encontrar patrones afines, los cuales se pueden clasificar en cuatro grupos así, no necesariamente exhaustivos: el $72 \%$ lo relaciona con una aproximación al análisis de la realidad social, un $43 \%$ con la incorporación del diálogo como búsqueda de soluciones, el 37\% opina que refleja aprender lo que se hace utilizando laboratorios y otros medios y un $51 \%$ lo relaciona con el trabajo sustentado.

\section{Conclusiones}

El ser humano es un ser que vive tantas dimensiones en su existencia como dimensiones sean generadas con el vivir en el lenguaje de los demás lo que le hace portador de un carácter social, afectivo, cultural, emocional, cognitivo y demás manifestaciones de la cultura que manifiesta con su conversación, luego su educación debe fundamentarse en el carácter humanista de los alumnos facilitando el diálogo entre compañeros y con el docente para incentivar la crítica y la proyección de su conocimiento hacia la transformación de su entorno .

El estudiante es un ser singular, en proceso permanente de formación, pero también es un ser social, lo cual implica que su estado actual es el resultado de lo previamente vivido por él en su entorno educativo, familiar, social y cultural; estado que no sólo está determinado por la razón, la inteligencia y la cultura, sino también por su capacidad comunicativa; mediante el uso de la comunicación comparte su ser con otros y se responsabiliza de cumplir las expectativas que los otros tienen de él; es un ser que se realiza a través de la convivencia dialogante con otros; es susceptible de ser formado en condiciones de libertad y autonomía; sus condiciones culturales permiten la formación de su conciencia crítica y por competencias que le habiliten para un mejor ser, hacer, saber hacer y convivir, en consecuencia de los parámetros de formación actuales.

La pedagogía liberadora sienta las bases de una nueva pedagogía opuesta a la tradicional, busca la reflexión y el cambio de las relaciones del individuo con la naturaleza y con la sociedad. Al contrario de la tradicional que postula un modelo de adaptación, la pedagogía de Freire es una "pedagogía del oprimido" que propone un modelo de ruptura, de cambio, de transformación total. La prioridad del docente es ayudar al alumno a lograr un punto de vista cada vez más crítico de su realidad a través del diálogo, donde ambos aprenden, como estímulo constante a la creatividad y al descubrimiento.

La docencia en la UFPS no es una actividad reducida a la enseñanza en el aula, es todo un sistema que a su vez está constituido por una serie de subsistemas, que interactúan para contribuir al mejoramiento continuo de su calidad y productividad en un proceso continuo de apropiación por parte de los docentes del enfoque pedagógico dialógicocrítico en los procesos de formación de sus estudiantes.

\section{Referencias}

Adorno, Th. 1966, [1984]. "Dialéctica Negativa”. Madrid: Taurus.

Areyuna, B. y V. Ilieva. (sf.). "Modernidad y Educación. Universidad" ARCIS y Fundación Universitaria del Área Andina.

Bakhtin, M. (1981). "The dialogic imagination: Four essays". Austin: University of Texas Press.

Calvo Martínez, T. (1986). "De los Sofistas a Platón: Política y Pensamiento". Madrid: Cincel.

Cabedo, S. (2000). "Fundamentos 
antropológicos para el diálogo transcultural: la ética comunicativa de Ludwig Feuerbach". Fòrum de Recerca. No. 5. Publicacions de L'uji.

Cervantes, L. (2009). "Pedagogía Crítica". http://www.slideshare.net/linacervantes/ pedagogia-critica-2042130

De Zubiría, J. (1994). "Tratado de Pedagogía Conceptual”. Los Modelos Pedagógicos. Bogotá: Fondo de Publicaciones Bernardo Herrera Merino.

De Zubiría, J. (2006). "Hacia una Pedagogía Dialogante". Los Modelos Pedagógicos. Cooperativa Editorial del Magisterio. Bogotá.

De Zubiría, J. (2007). "Modelos Pedagógicos Contemporáneos". Cooperativa Editorial del Magisterio. Bogotá.

Díaz, M. (2003). "abc. Modelos Educativos Pedagógicos y Didácticos". Vol. I. Ediciones SEM Servicios Editoriales del Magisterio. Heladio Moreno M. Compilación. 2ª Ed. Bogotá.

Díaz, M. (2009). "Modelo Pedagógico Integrado". Medellín: Universidad Pontificia Bolivariana.

Díez, F. (2000). "La Enseñanza de las Matemáticas en la Educación de Personas Adultas, un Modelo Dialógico". Facultad de Pedagogía. Universidad de Barcelona.

Ferrada, D y F. Ramón. (2008). "El Modelo Dialógico de la Pedagogía: Un Aporte desde las Experiencias de Comunidades de Aprendizaje". Estudios Pedagógicos XXXIV, (1), pp. 41-61.

Feuerbach, L. (1983). "Principios de la filosofía del futuro", Barberá del Vallés, Humanitas.

Flórez Ochoa, R. (2005). "Pedagogía del conocimiento". Madrid: McGraw Hill.

Freire, P. (1970). "Pedagogía del Oprimido". Madrid: Siglo XXI.

Freire, P. (1979). "Pedagogía del Oprimido".
Madrid: Siglo XXI.

Freire, P. (1990). "La Naturaleza política de la educación. Cultura, poder y liberación". España: Ediciones Paidós.

Gallardo, H. (2013). "Calidad y Productividad de la Docencia en la Universidad Francisco de Paula Santander". Disertación de Ph. D. Universidad Pedagógica Experimental Libertador. Rubio (Ven.).

Giroux, H and P. McLaren. (1989). "Critical Pedagogy, the State, and the Struggle for Culture". New York: State University Press of New York.

Góngora, J. (2008). "Desarrollo del Vínculo Docencia-Investigación en la Formación Académica". Disertación de Ph. D. Instituto Humanista de Sinaloa.

Habermas, J. (1987). "Teoría de la Acción Comunicativa". Madrid: Ed. Taurus.

Horkheimer, M. (2003). "Teoría Crítica". 1a ed. 3 r reimp. Buenos Aires: Amorrortu.

Kemmis, S., P. Cole and D. Suggett. (2007). "Hacia una Escuela Socialmente Crítica". Linterna Pedagógica. Nau LLibres.

Marcuse, H. (1954) [1993]. "El Hombre Unidimensional”. Boston: Bacon Press.

Marx, K. (1867) [1998]. "El Capital. México. Siglo XXI.

McLaren, P. (1995). "Critical Pedagogy and Predatory Culture: Oppositional Politics in a Postmodern Era", New York \& London: Routledge.

Miraval, A. (2008). "Pedagogía Crítica: Algunos Componentes Teóricometodológicos". En M. Gadotti (Comp.) Paulo Freire Contribuciones para la Pedagogía.

Not, L. (1962). "La Enseñanza Dialogante". Barcelona: Herder.

Platón. (1972). “Obras”. Madrid: Aguilar. 
No. 2

Julio-Diciembre 2014

ISSN 0122-820X

PP: 81-92

Sen, A. (2007). "India Contemporánea: entre la modernidad y la tradición”. Barcelona: Gedisa.

Soler, M. (2004). "Reading to share: Accounting for others in dialogic literary gatherings". Aspects of the Dialogic Self, p.p. 157-183. Berlín: Lehmans.

UFPS. (2007). "Proyecto Educativo Institucional". Universidad Francisco de Paula Santander. Cúcuta, Colombia. 2007

Urbina, J. (2010). "El enfoque pedagógico de la ufps: la perspectiva crítica y dialógica como posibilidad para el mejoramiento de la docencia universitaria”. Cúcuta: UFPS.

Weber, M. (1996). "Economía y Sociedad". México: Fondo de Cultura Económica.

Wells, G. (2001). "Indagación Dialógica: hacia una teoría y una práctica socioculturales en la educación”. Barcelona: Paidos. 